\title{
Effect of Error Packetization on the Quality of Streaming Video in Wireless Broadband Networks
}

\author{
Aderemi A. Atayero \\ Department of Electrical and Information Engineering \\ Covenant University \\ Ota, Nigeria
}

\begin{abstract}
A Markov model describing the duration of error intervals and error-free reception for streaming video transmission was developed based on the experimental data obtained as a result of streaming video from a mobile source on IEEE 802.16 standard network. The analysis of experimental results shows that the average quality of video sequences when simulating Markov model of packetization of errors are similar to those obtained when simulating single packet errors with PER index in the range of $3 \times 10^{-3}$ to $1 \times 10^{-2}$. An algorithm for creating software for simulating packetization of errors was developed. In this paper we describe the algorithm, software developed based on this algorithm as well as the Markov model created for the modeling.
\end{abstract}

Keywords-Video streaming; Markov model; IEEE 802.16; Bit Error Rate; Burst Error Length; Packet Error Rate; Codec.

\section{INTRODUCTION}

The need to create realistic simulation and mathematical models of behavior of losses in the communication channels based on the apparatus of Markov chains for wireless access systems is a scientific problem of important consequence. Markov processes with the necessary number of states sufficiently describe the mechanism of transmission of information [1], the knowledge of which is necessary to analyze network problems during packet video transmission. The parameters of the model make it possible to determine the quality of transmitted video as well as the statistical parameters of the network.

A model decscribing the length of error intervals and errorfree reception for streaming video transmission was developed based on the experimental data obtained as a result of streaming video from a moving source on WiMAX network [2]. Based on the graph of packet loss distribution, an array was formed in which the lost packet corresponds to a logic zero (0) and received packet corresponds to a logic unit (1). The original array was split into two, one of which contains information about the lost packets and the other contains information about the received packets. The formation of arrays was carried out in accordance with the procedure shown in Figure 1.

\author{
Oleg I. Sheluhin and Yury A. Ivanov \\ Department of Information Security \\ Moscow Tech. Univ. of Communication and Informatics \\ Moscow, Russia
}

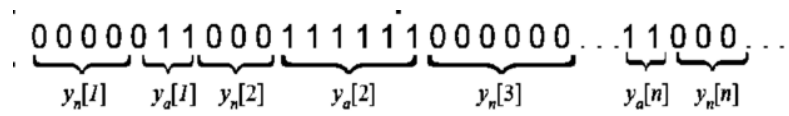

Figure 1. Formation of Arrays

\section{MARKov Model DesCribing The EXPERIMENTAL DATA}

In accordance with the method presented in [3], the available raw data file was divided into two parts, each of which separately contains the duration of ON periods and OFF periods. Variables $\mathrm{y}_{\mathrm{a}}[\mathrm{n}]$ fall under the ON periods, while variables $\mathrm{y}_{\mathrm{n}}[\mathrm{n}]$ fall under the OFF peroids. An approximation of the distribution function (DF) of real processes is obtained. Equation (1) is used for approximating the distribution function of OFF state.

$$
F^{*}(k)=A_{i} \sum_{i=1}^{3} e^{-\alpha_{i} k}
$$

By using the method of least squares we find the unknown coefficients of the approximation for the expression (1) as presented in Table 1.

TABLE I. APPROXIMATION COEFFicient VAlues $\left(A_{1} ; A_{1}\right)$

\begin{tabular}{|c|c|c|c|c|c|}
\hline $\boldsymbol{A}_{\mathbf{1}}$ & $\boldsymbol{\alpha}_{\mathbf{1}}$ & $\boldsymbol{A}_{\mathbf{2}}$ & $\boldsymbol{\alpha}_{\mathbf{2}}$ & $\boldsymbol{A}_{\mathbf{3}}$ & $\boldsymbol{\alpha}_{\mathbf{3}}$ \\
\hline 0.612086 & 0.072672 & 0.631933 & 0.540023 & 0.073586 & 0.040006 \\
\hline
\end{tabular}

Substituting the coefficient values obtained and given in Table 1 into equation (1), we obtain the approximation of the original distribution of the length of OFF periods as equation (2):

$=0.612086 \times e^{-0.072672 k}+0.631933 \times e^{-0.540023 k}$

$+0.073586 \times e^{-0.040006 k}$

Equation (3) is used for approximating the distribution function of ON state. 


$$
F^{*}(k)=B_{i} \sum_{i=1}^{6} e^{-\beta_{i} k}
$$

The unknown coefficients of the approximation for the expression (3) are found using the method of least squares and presented in Table 2.

TABLE II. APPROXIMATION COEFFICIENTS $\left(\mathrm{B}_{1} ; \mathrm{B}_{\mathrm{I}}\right)$

\begin{tabular}{|c|c|c|c|c|c|}
\hline $\boldsymbol{B}_{\mathbf{1}}$ & $\boldsymbol{\beta}_{\mathbf{1}}$ & $\boldsymbol{B}_{\mathbf{2}}$ & $\boldsymbol{\beta}_{\mathbf{2}}$ & $\boldsymbol{B}_{\mathbf{3}}$ & $\boldsymbol{\beta}_{\mathbf{3}}$ \\
\hline 0.065836 & 0.000643 & 0.107716 & 0.000708 & 0.33109 & 0.007203 \\
\hline $\boldsymbol{B}_{\mathbf{4}}$ & $\boldsymbol{\beta}_{\mathbf{4}}$ & $\boldsymbol{B}_{\mathbf{5}}$ & $\boldsymbol{\beta}_{\mathbf{5}}$ & $\boldsymbol{B}_{\mathbf{6}}$ & $\boldsymbol{\beta}_{\mathbf{6}}$ \\
\hline 0.057449 & 0.0000618 & 0.007203 & 0.291568 & 0.224767 & 0.0094038 \\
\hline
\end{tabular}

Substituting the coefficient values obtained and given in Table 2 into equation (3), we obtain the approximation of the original distribution of the length of $\mathrm{ON}$ periods as equation (4):

$$
\begin{array}{r}
F^{*}(k)=0.065836 \times e^{-0.000643 k}+0.107716 \times \\
e^{-0.000708 k}+0.33109 \times e^{-0.007203 k}+0.057449 \times \\
e^{-0.0000618 k}+0.007203 \times e^{-0.291568 k}+0.224767 \times \\
e^{-0.0094038 k}
\end{array}
$$

The approximation of DF of ON is shown in Figure 2.

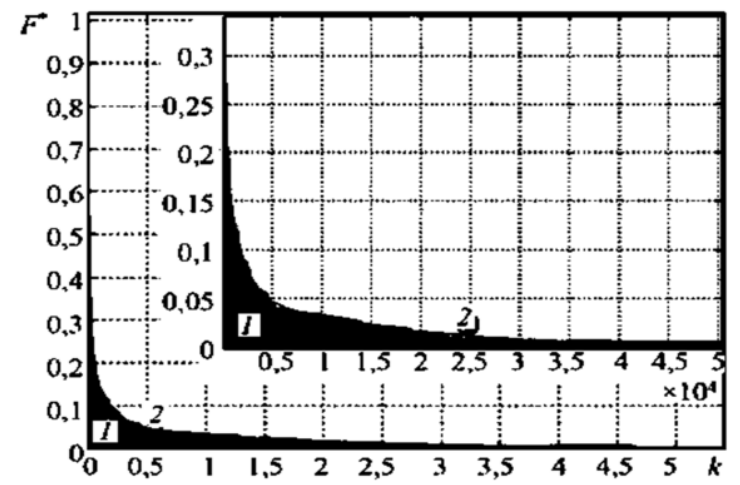

Figure 3. DF of ON approximation (2), DF of ON experiment (1). (embedded graph - reduced scale of DF of ON)

\begin{tabular}{|c|c|c|c|c|c|c|}
\hline & $A_{1}$ & $A_{2}$ & $A_{3}$ & $B_{1}$ & $B_{2}$ & $B_{3}$ \\
\hline$A_{1}$ & $e^{-\alpha 1}$ & 0 & 0 & $\left(1-e^{-\alpha 1}\right) B_{1}$ & $\left(1-e^{-a l}\right) B_{2}$ & $\left(1-e^{-\alpha 1}\right) B$ \\
\hline$A_{2}$ & 0 & $e^{-\alpha 2}$ & 0 & $\left(1-e^{-\alpha 2}\right) B_{1}$ & $\left(1-e^{-a 2}\right) B_{2}$ & $\left(1-e^{-\alpha 2}\right) B_{3}$ \\
\hline & 0 & 0 & $e^{-\alpha 3}$ & $\left(1-e^{-a 3}\right) B_{1}$ & $\left(1-e^{-\alpha 3}\right) B_{2}$ & $\left(1-e^{-\alpha 3}\right) B_{3}$ \\
\hline & $\left.\left.-e^{-\beta \mid}\right)\right) A_{1}$ & $\left(1-e^{-\beta 1}\right) A_{2}$ & $\left(1-e^{-\beta 1}\right) A_{3}$ & $e^{-\beta 1}$ & 0 & 0 \\
\hline & $\left.-e^{-\beta 2}\right) A_{1}$ & $\left(1-e^{-\beta 2}\right) A_{2}$ & $\left(1-e^{-\beta 2}\right) A_{3}$ & 0 & $e^{-\beta 2}$ & 0 \\
\hline & $\left.-e^{-\beta 3}\right) A_{1}$ & $\left(1-e^{-\beta 3}\right) A_{2}$ & $\left(1-e^{-\beta 3}\right) A_{3}$ & 0 & 0 & $e^{-\beta 3}$ \\
\hline
\end{tabular}

After the normalization of obtained approximating expressions (2) and (4), additional distributions of duration of $\mathrm{ON}$-and OFF-processes, the matrix of transition probabilities are created, which is of the form presented in Figure 3:

Figure 4. The matrix of transition probabilities
Substituting the values of the coefficients found in Tables 1 and 2 into the matrix of transition probabilities, we obtain the matrix of values in Figure 4.

$$
\Gamma=\left|\begin{array}{cccccc}
0.999 & 0 & 0 & 2.4 * 10^{-5} & 8.69 * 10^{-4} & 1.1 * 10^{-4} \\
0 & 0.9944 & 0 & 1.344 * 10^{-4} & 0.0049 & 6.16^{*} 10^{-4} \\
0 & 0 & 0.965 & 8.4 * 10^{-4} & 0.0304 & 0.0039 \\
1.8^{*} 10^{-5} & 3.6^{*} 10^{-4} & 3.6 * 10^{-4} & 0.9991 & 0 & 0 \\
4.2 * 10^{-5} & 8.4 * 10^{-4} & 8.4 * 10^{-4} & 0 & 0.9979 & 0 \\
2.58 * 10^{-4} & 0.0052 & 0.0052 & 0 & 0 & 0.9871
\end{array}\right|
$$

Figure 2. The matrix of values

\section{SOFTWARE For ERROR PACKETIZATION SimUlation}

Simulation of the transmission of streaming video traffic over a WiMAX network can be done given the probability transition matrix and vector of initial probabilities [4]. The choice of the initial state of the system was carried out using the condition that all states are equiprobable (i.e. $\mathrm{p}=$ $1 / \mathrm{N}$, where $\mathrm{N}$-number of states the system can be in after DF approximation). Description of the block diagram of the simulation algorithm is as given below, while the Markov model is shown in Figure 5.

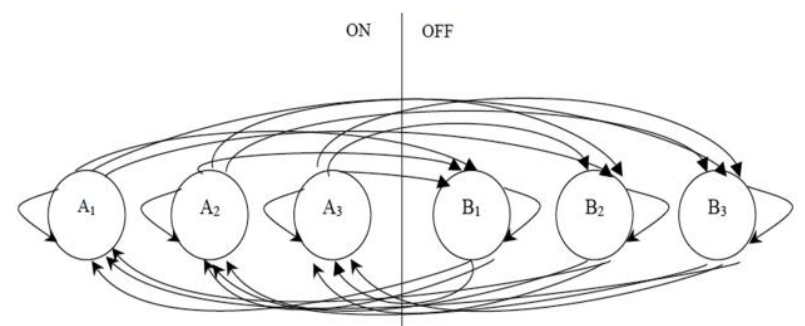

Figure 5. Markov model of error packetization algorithm

\section{DESCRIPTION OF ERROR PACKETIZATION AlgORITHM}

STEP 1. Start program (Description of the variables, functions, procedures and modules used)

STEP 2. Enter two-dimensional array matrix of transition probabilities. In the developed software, this matrix was given as an array of constants in the declarations section and named markov.

STEP 3. Set state from which to begin modeling. Since a 9state model was chosen, the state variable can take integer values on the interval $(1-9)$. Also, at this stage of the algorithm the accumulated variables summa_on and summa_off, which reflect the duration of the ON periods and OFF periods, are reset to zero respectively.

STEP 4. Begin cycle with parameter $i$. The number of iterations equals the number of transitions in the simulated system.

STEP 5. Instantiate the built-in generator of pseudorandom uniformly distributed sequence, generating a random value in the interval $(0,1)$. Assign the generated value to rnd. At the moment of generating the variable $r n d$, the system moves to the next state. The 
exact state into which it falls will be determined by the subsequent actions of the algorithm. The variable summa is reset to zero.

STEP 6. Start the cycle with parameter $k$. The number of iterations in the cycle equals the number of states of the system being modeled. For this case, the number of iterations is eight (8). This loop is used to determine the state into which of the system has moved at the particular time of consideration.

STEP 7. Check - does the value of $r n d$ fall in the $k^{\text {th }}$ state of the Markov chain. At the same time the following variables are involved: summa - accumulates the probability of all states up to the $k^{\text {th }}$; markov [state, $\mathrm{k}$ ] - a two-dimensional array, which contains the transition matrix. If rnd falls within a range of probabilities corresponding to the $k^{\text {th }}$ state, then goto step 8, otherwise goto step 9 .

STEP 8. Check - in which state is the process currently? If in the active state, then goto step 10. If in passive state, then goto step 11 .

STEP 9. The summa variable is increased by the value of the probability of being in state $\mathrm{k}$. Then proceed to the next iteration of step 6 .

STEP 10. Check - was the last state of the matrix passive? If yes, goto step 12. Otherwise, goto step 16.

STEP 11. Check - was the last state of the matrix active? If yes, goto step 13. Otherwise, goto step 17

STEP 12. Arrival at this step implies the end of OFF period. Therefore save or print to file summa_off.

STEP 13. Arrival at this step implies the end of ON period. Therefore save or print to file summa_on.

STEP 14. Since the OFF period as ended, reset the variable summa_off to zero in preparation for the record of fresh OFF-period information, when the process will be in the passive state.

STEP 15. Since the ON period as ended, reset the variable summa_on to zero in preparation for the record of fresh ON-period information, when the process will be in the active state.

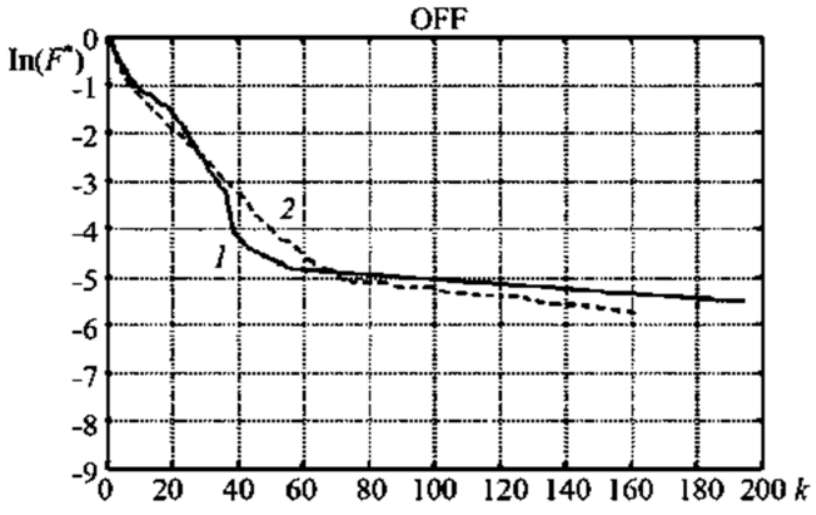

a)
STEP 16. Arrival at this step implies either the continuation of the previous ON period, or the start of a new ON period. So increment the variable summa_on and assign the value of cycle $k$ to the state variable.

STEP 17. Arrival at this step implies either the continuation of the previous OFF period, or the start of a new OFF period. So increment the variable summa_off and assign the value of cycle $k$ to the state variable.

STEP 18. At this step of the algorithm, the system just transited to the next state, so turn to the next iteration of the parameter $i$.

STEP 19. End program.

As a result, the amount of packets falling either in the received state or the lost state in a row is accumulated (summa_ON $=$ summa_ON +1$)$.

\section{DISCUSSION}

Distribution function of $\mathrm{ON}$ - and OFF-processes for both the simulated and experimental sequences was obtained using the described Markov model shown in Figure 6.

Experiments show that increasing the number of states of the Markov model describing the packetization of errors allows for obtaining a satisfactory correspondence between the results of the experimental data and the data obtained by simulation.

\section{A. Markov Model of Packetization of Errors}

Two independent datasets, each containing 300,000 values were generated with the aid of the developed Markov model [5]. This amount of data allows for a qualitative comparison of RTP packets from the experiment conducted on the transmission of a 30-minute streaming video on a real WiMAX network [2] with the results of the experiment conducted using the HSC.

In the model, each value in the array is represented by the numbers 0 or 1 , where 0 means error-free value, and 1 erroneous value. Figure 7 shows the distribution of data set values, where the white areas correspond to error-free values (0), and black - erroneous values (1).

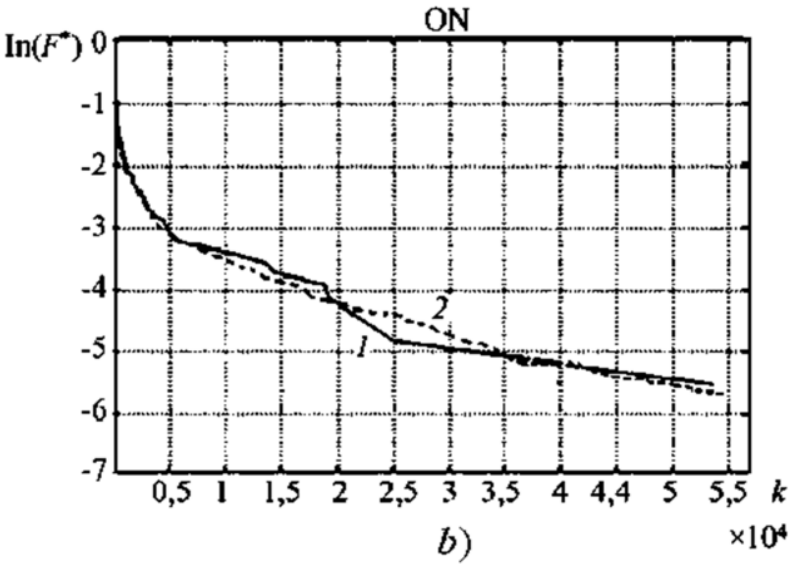

Figure 6. DF of simulated samples of the length of OFF-(a) and ON (b) - periods: curve 1 - experiment, curve 2 - simulation 

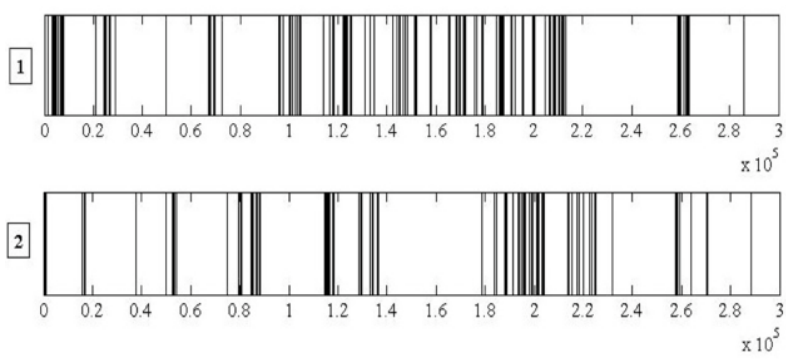

Figure 7. Distribution of error-free and erroneous values for arrays №1 and №2.

The first array contains $2,743(0.91 \%)$, and the second has $2,430(0.81 \%)$ errorneous values. The distribution of the number of errors in the same error group is presented in the form of histograms in Figure 8.
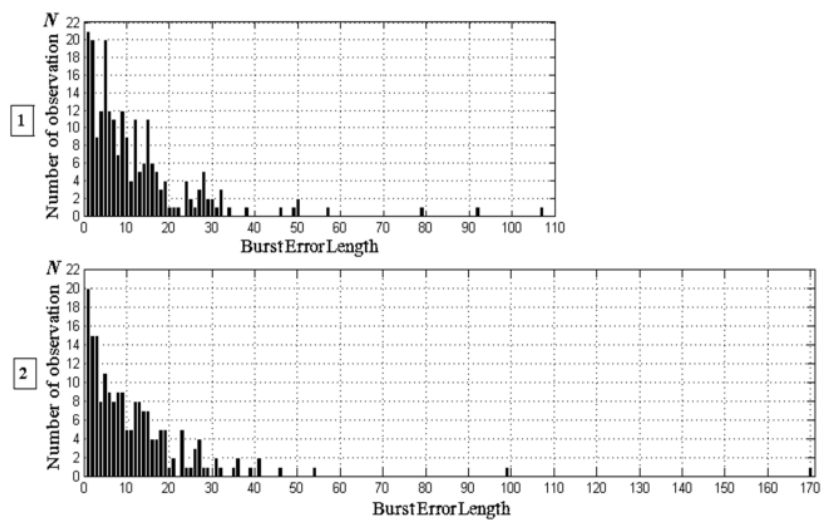

Figure 9. Distribution of errors in a group of bugs array №1 and №2.

It is shown that the distribution of errors cannot be approximated by an exponential function, a fact that validates the use of the Markov model. Furthermore, in order to study the influence of the Markov model of packetization of errors on the quality of video streaming, simulation of the transmission of a 30-minute video in the structure of the HSC was conducted. The simulation entailed the transmission and reception of traces over an "ideal" channel with unlimited bandwidth and no delay in the NS-2 environment [6]. Subsequently each packet of the receive trace was matched with a corresponding value from the dataset array (packet id $=$ serial value of the data set array). All packets corresponding to 1 (indicating error) were deleted. This allowed for simulating sequence of errors that occur in the network and to effect corrective decoding of the video stream.

Two experiments were carried out with arrays №1 and №2 respectively. Figure 9 shows a block diagram of the experiments. The results of experimental quality indicators obtained are shown in Figures 10-12.

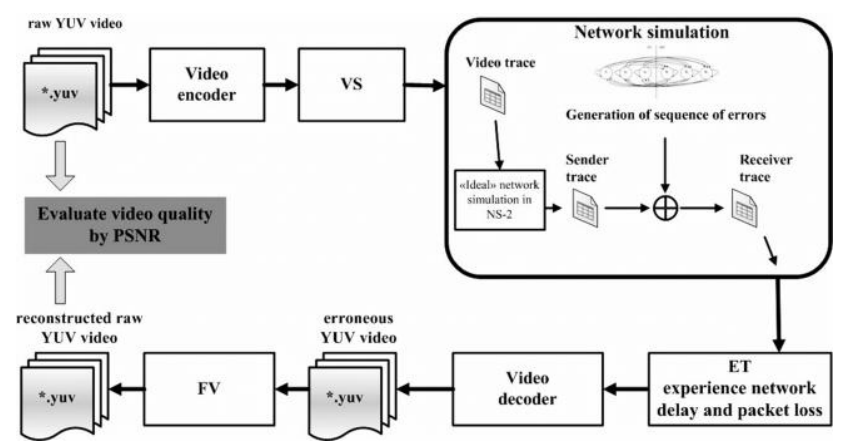

Figure 8. Block diagram of the experiments №1 and №2.
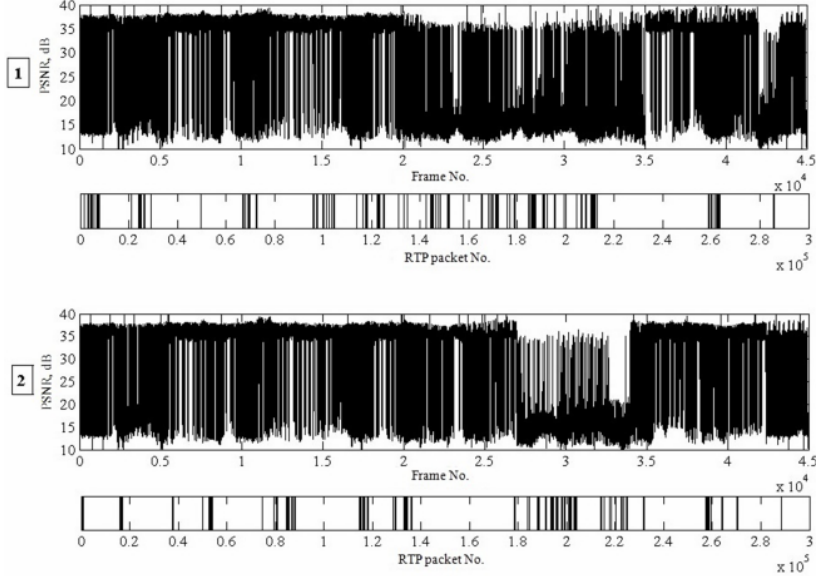

Figure 10. The change the PSNR indicator from experiments №1 and
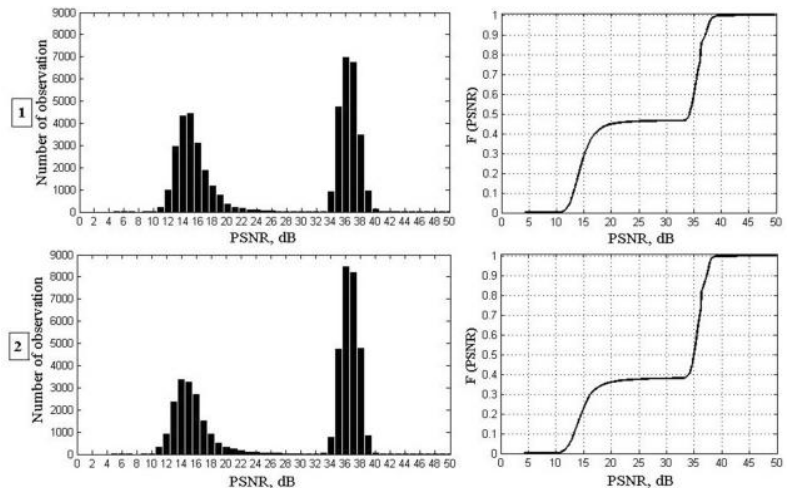

Figure 11. Histogram and distribution function of the PSNR indicator in experiments №1 and №2 


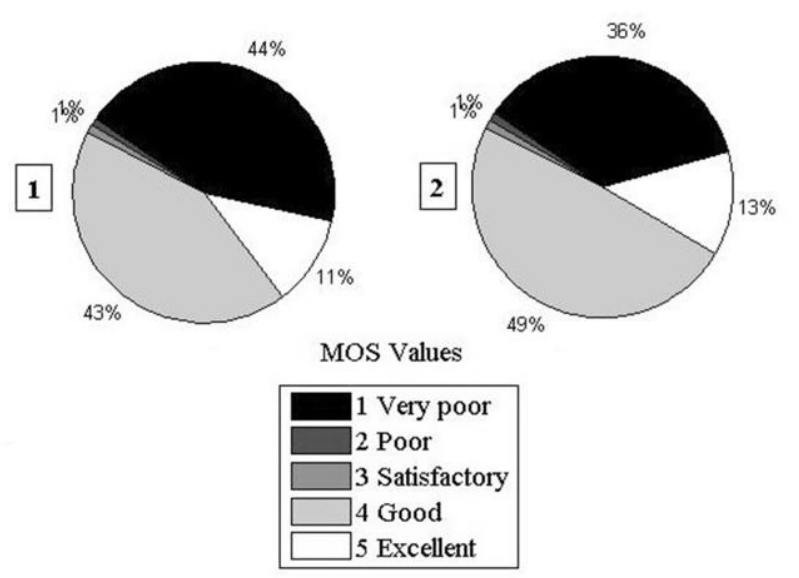

Figure 12. MOS Quality value for video broadcast in №1 and №2

\section{CONCLUSION}

Analysis of the quality of received video sequence when simulating Markov model of error packetization shows that the average quality of video sequences is slightly worse than during transmission over a real network. For example, in an experiment on streaming video over a real WiMAX network, the average quality of $31 \mathrm{~dB}$ was obtained, and for the simulations $26 \mathrm{~dB}$ and $28 \mathrm{~dB}$ respectively. The subjective MOS quality indicator also shows a difference in values: a real WiMAX network returned a mean value of 3.59 (corresponding to satisfactory), while the experiments returned values of 2.72 (corresponding to poor) and 3.01 (corresponding to satisfactory), respectively. This suggests that the Markov model of packetization of error obtained from a real network for streaming video can be used in the simulaton of transmission of video across networks in the hardware-software complex developed by the authors in a previous work [7].

The average quality of video sequences obtained from simulations of the Markov model are similar to those obtained when simulating single packet errors with PER index in the range of $3 \times 10^{-3}$ to $1 \times 10^{-2}$. While the length of error group depending on the PER of the specified range can attain values of $\mathrm{BEL} \leq 10$.

\section{REFERENCES}

[1] H. Wang and N. Moayeri, "Finite state Markov channel - a useful model for radio communication channels," IEEE Trans. on Vehicular technology, vol. 44, № 2, pp. 163-171, February 1995.

[2] Atayero A.A., Sheluhin O.I., Ivanov Y.A. and Iruemi J.O., "Effect of wideband wireless access systems interference robustness on the quality of video streaming," Lecture Notes in Engineering and Computer Science: Proceedings of The World Congress on Engineering and Computer Science 2011, WCECS 2011, 19-21 October, 2011, San Francisco, USA, pp. 848-854.
[3] ITU P.800: Methods for subjective determination of transmission quality, available at: http://www.itu.int/rec/T-REC-P.800-199608-I/en.

[4] Deb S., Jaiswal S. and Nagaraj K., "Real-time video multicast in WiMAX networks," Proc. of IEEE INFOCOM, April 2008.

[5] Hohlfeld O., "Markovian packet loss generators and video QoE, T Systems, February 2008.

[6] NS-2 Documentation, Available at: http://www.isi.edu/nsnam/ns/nsdocumentation.html, last accessed 11/11/2011.

[7] A.A. Atayero, O.I. Sheluhin, Y.A. Ivanov, A.S. Alatishe "Estimation of the Visual Quality of Video Streaming Under Desynchronization Conditions," International Journal of Advanced Computer Science and Applications (IJACSA), vol. 2, № 12, pp. 1-11, December 2011.

\section{AUTHORS PROFILE}

Aderemi A. Atayero graduated from the Moscow Institute of Technology (MIT) with a B.Sc. Degree in Radio Engineering and M.Sc. Degree in Satellite Communication Systems in 1992 and 1994 respectively. He earned a $\mathrm{PhD}$ in Telecommunication Engineering/Signal Processing from Moscow State Technical University of Civil Aviation, Russia in 2000.

$\mathrm{He}$ is a member of a number of professional associations including: the Institute of Electrical and Electronic Engineers, IEEE, the International Association of Engineers, IAENG, and a professional member of the International Who's Who Historical Society (IWWHS) among others. He is a registered engineer with the Council for the Regulation of Engineering in Nigeria, COREN. A two-time Head, Department of electrical and Information Engineering, Covenant University, Nigeria, he was the coordinator of the School of Engineering of the same University.

Dr. Atayero is widely published in International peer-reviewed journals, proceedings, and edited books. He is on the editorial board of a number of highly reputed International journals. Atayero is a recipient of several awards including the '2009/10 Ford Foundation Teaching Innovation Award'. His current research interests are in Radio and Telecommunication Systems and Devices; Signal Processing and Converged Multi-service Networks.

Oleg I. Sheluhin was born in Moscow, Russia in 1952. He obtained a M.Sc. Degree in Radio Engineering1974 from the Moscow Institute of Transport Engineers (MITE). He later enrolled at Lomonosov State University (Moscow) and graduated in 1979 with a Second M.Sc. in Mathematics. He received a PhD at MITE in 1979 in Radio Engineering and earned a D.Sc. Degree in Telecommunication Systems and Devices from Kharkov Aviation Institute in 1990. The title of his $\mathrm{PhD}$ thesis was 'Investigation of interfering factors influence on the structure and activity of noise short-range radar'.

$\mathrm{He}$ is currently Head, Department of Information Security, Moscow Technical University of Communication and Informatics, Russia. He was the Head, Radio Engineering and Radio Systems Department of Moscow State Technical University of Service (MSTUS).

Prof. Sheluhin is a member of the International Academy of Sciences of Higher Educational Institutions. He has published over 15 scientific books and textbooks for universities and has more than 250 scientific papers. He is the Chief Editor of the scientific journal Electrical and Informational Complexes and Systems and a member of Editorial Boards of various scientific journals. In 2004 the Russian President awarded him the honorary title 'Honored Scientific Worker of the Russian Federation'.

Yury A. Ivanov was born in Moscow, Russia in 1985. He obtained a M.Sc. degree in Systems, network and devices in telecommunications from Chuvash State University in 2007. He obtained a Ph.D in Telecommunication Networks and Systems in 2011 from Moscow State University of Communication and Informatics. Dr. Ivanov has published over 35 scientific papers and his current research interests include Radio and Telecommunications Systems and Devices: transmission of multimedia data across telecommunication networks, assessment of the quality of video sequences. Dr. Ivanov is a member of a number of professional associations including: the Institute of Electrical and Electronic Engineers, IEEE, the International Association of Engineers, IAENG. 